\title{
Global and local level dynamics in chaotic microwave billiards
}

\author{
M. Barth, U. Kuhl, and H.-J. Stöckmann
}

Fachbereich Physik, Philipps-Universität Marburg, Renthof 5, D-35032 Marburg, Germany

michael.barth@physik. uni-marburg.de

Received dd.mm.yyyy, accepted dd.mm.yyyy by xx

Abstract. The spectra of Sinai microwave billiards and rectangular billiards with statistically distributed circular scatterers have been taken as a function of the position of one wall, and of one of the scatterers, respectively. Whereas in the first case the velocity distribution and correlations obey the universal behaviour predicted by Simons and Altshuler, in the second case a completely different behaviour is observed. This is due to the fact that a shift of one wall changes the wave function globally whereas the displacement of one scatterer only leads to a local perturbance.

Keywords: Universal velocity correlations, mesoscopic systems, microwave billiards PACS: 05.45.-a, 73.23.-b

\section{Introduction}

The eigenvalue dynamics of the spectra of disordered systems in dependence of an external parameter has attracted considerable interest in recent years. It has been motivated by the fact that the conductance through a system is closely linked to the sensitivity of its eigenvalues on a perturbation [1, 2]. In this respect the work of Akkermans and Montambaux has to be mentioned in particular [3], who showed that the conductance can be expressed in terms of the quadratically averaged eigenvalue velocities,

$$
C \sim\left\langle\left|\frac{\partial E_{n}}{\partial X}\right|^{2}\right\rangle .
$$

Here the brackets denote a local average, and $X$ is the external parameter. Later it was shown by Simons and Altshuler using supersymmetry techniques that the eigenvalue velocities $v$ should be Gaussian distributed [5]

$$
P(v)=\frac{1}{\sqrt{2 \pi\left\langle v^{2}\right\rangle}} \exp \left(-\frac{v^{2}}{2\left\langle v^{2}\right\rangle}\right) .
$$

Moreover, if the eigenvalues $E_{n}$ and the level dynamics parameter $X$ are rescaled according to

$$
\epsilon_{n}=\frac{E_{n}}{\Delta}, \quad x=\frac{1}{\Delta} \sqrt{\left\langle\left|\frac{\partial E_{n}}{\partial X}\right|^{2}\right\rangle} X,
$$



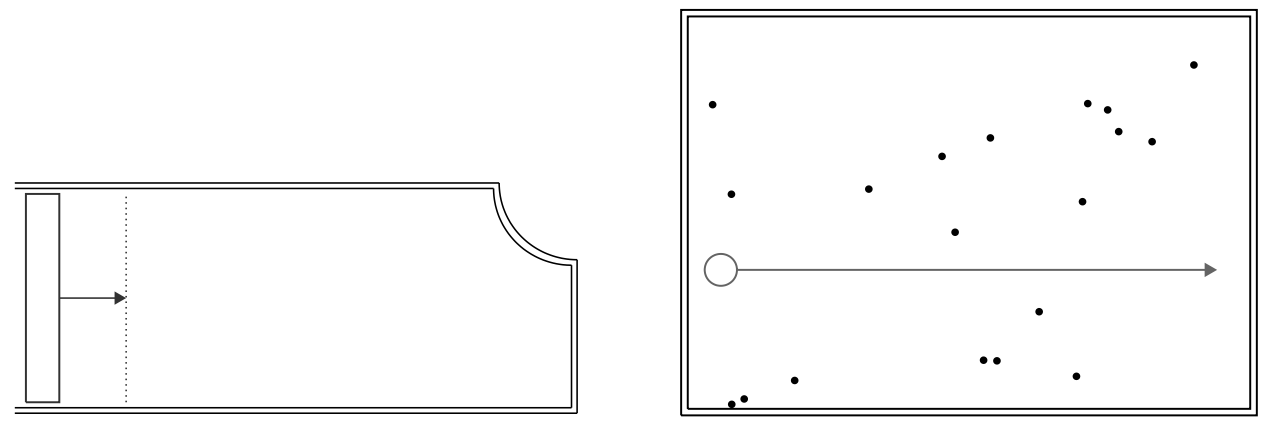

Fig. 1 Sketch of the quarter Sinai billiard used for the global level dynamics (left) and of the rectangular billiard with randomly distributed scatterers (right). Both figures are drawn in scale, including size and positions of the scatterers. For the dimensions see the text.

where $\Delta$ is the mean level spacing, the velocity autocorrelation function

$$
c(x)=\left\langle\frac{\partial \epsilon_{n}\left(x_{0}+x\right)}{\partial x_{0}} \cdot \frac{\partial \epsilon_{n}\left(x_{0}\right)}{\partial x_{0}}\right\rangle-\left\langle\frac{\partial \epsilon_{n}\left(x_{0}\right)}{\partial x_{0}}\right\rangle^{2}
$$

should obey a universal behaviour 国, 战. A recent review on these questions can be found in $\| 6]$.

There are a number of attempts to study the autocorrelation function (4), both theoretically and experimentally. The systems under consideration ranged from the hydrogen atom in a strong magnetic field [7] via conformally deformed [8] and raysplitting billiards [9] to the acoustic spectra of vibrating quartz blocks [10]. In all cases the overall behaviour predicted by Simons and Altshuler was more or less observed but in nearly all cases there had been significant deviations as well.

This was our motivation to study velocity distributions and correlations in various mircowave billiards by applying different types of parameter variations. Most to our surprise we found that the velocity distributions show a clear dependence on the type of parameter variation. Only for the type classified as 'global' below, we found Simons' and Altshuler's universal behaviour, whereas for a second type denoted 'local' a completely different behaviour was found. A preliminary version of this paper has been published already in [11].

\section{Experiment}

All results presented below have been obtained by taking the eigenfrequency spectra of billiard-shaped microwave cavities, shortly termed microwave billiards. The technique is described in [12]. Here it may be sufficient to note that in quasi-two-dimensional resonators, i.e. cavities with top and bottom plate parallel to each other, quantum mechanical and electromagnetic spectra are completely equivalent, as long as the maximum frequency $\nu_{\max }=c / 2 h$ is not surpassed, where $h$ is the height of the resonator. In the present experiment the height was $8 \mathrm{~mm}$ yielding a maximum frequency of 18.74 $\mathrm{GHz}$. 

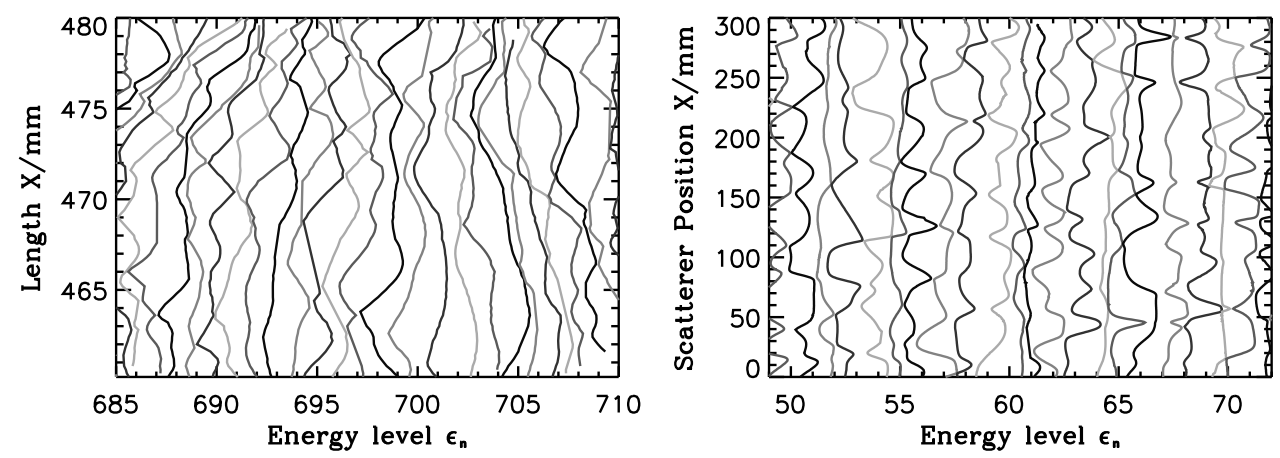

Fig. 2 Part of the eigenvalue level dynamics spectra for the quarter Sinai billiard as a function of the length (left) and the rectangular billiard with randomly distributed scatterers as a function of the position of one scatterers (right). All spectra have been unfolded to a mean density of one.

In the very beginning it was not evident at all which billiard parameter should be varied. According to Simons and Altshuler the parameter $X$ 'could denote the strength of some field, like the Aharonov-Bohm flux through a ring, or a magnetic field, or the position of some impurity' (cited literally from Ref. [5]). In microwave billiards the magnetic field is not available as a parameter (though the use of billiards with ferritecoated walls may offer an alternative [13]). On the other hand it is straightforward to study the spectra of microwave billiards as a function of some length [14]. The magnetic field and the length variation have in common that already a small change in the parameter will modify the wavefunction everywhere. For this reason we call this type of level dynamics 'global'. The variation of the position of one impurity, on the other hand, can be easily performed in microwave billiards, with the additional advantage that, in contrast to real mesoscopic systems, we can control the disorder in every moment perfectly. The displacement of one impurity changes the value of the wave function only in its neighbourhood. Consequently, we call this type of level dynamics 'local'.

Both types of level dynamics were realized in the experiments. One of the systems studied was a quarter Sinai billiard with width $b=200 \mathrm{~mm}$, radius $r=70 \mathrm{~mm}$ of the quarter circle, and a length $a$ which was varied between 460 and $480 \mathrm{~mm}$ in steps of $0.2 \mathrm{~mm}$. About 120 eigenvalues entered into the data analysis in the frequency range 14.5 to $15.5 \mathrm{GHz}$. The second system was a rectangular billiard with side lengths $a=340 \mathrm{~mm}, b=240 \mathrm{~mm}$, containing 19 circular disks with a diameter of $4.6 \mathrm{~mm}$ whose positions were determined by means of a random number generator. The position of one further disk was varied in one direction in steps of $1 \mathrm{~mm}$. Two different movable disks were used with diameters of 4.6 and $20 \mathrm{~mm}$, respectively (see Fig. 1).

A part of the level dynamic spectrum of the Sinai billiard is shown in Fig. 2 (left). As a function of length the resonances experience four to five avoided crossing in the studied length range. This is about the maximum range usable for the velocity correlation measurements for the following reason. Upon variation of the length it happens that a node line of an eigenfunction passes the position of the coupling antenna, 

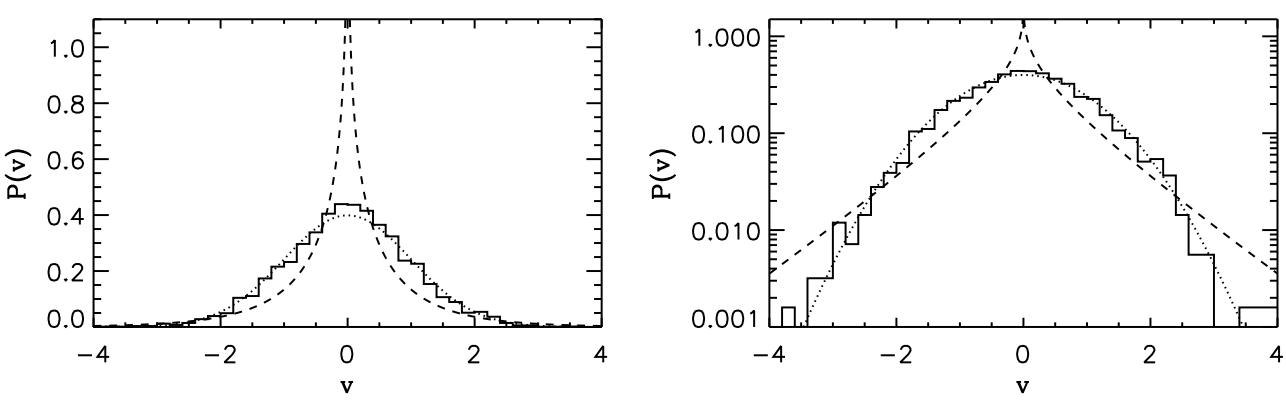

Fig. 3 Velocity distribution for the quarter Sinai billiard on a linear (left) and a logarithmic (right) scale. The dotted line corresponds to a Gaussian, the dashed line to a modified Bessel function (see Eq. (月)).

leading to a temporary loss of the eigenfrequency. In the studied length range each resonance disappeared at most once, but with increasing length range the tieing up of the open ends became more and more unreliable. The situation changes qualitatively of the level dynamics of the rectangular billiard with randomly distributed scatterers shown in Fig. 2 (right). Already a comparison by eyes shows that there is much more regularity in the latter case. Now it is no problem to follow each eigenvalue over about 10 avoided crossings.

\section{Global level dynamics}

We start with a discussion of the Sinai billiard results. Fig. 3 shows the found velocity distribution both on a linear and a logarithmic scale. The found distribution is close to a Gaussian in accordance with the universal prediction. The dashed line is a modified Bessel function which will become important in the following. It is well-known that non-generic features such as bouncing balls give rise to deviations from a Gaussian distribution in particular in the region of the wings [14, 15]. In the measurement we therefore carefully avoided the regions disturbed by the dominating bouncing ball. Hence such a disturbance does not show up in the present case.

Fig. 1 shows the corresponding velocity autocorrelation function. To obtain the result, each eigenvalue was followed over the available length range including four to five avoided crossings. The mean squared velocity needed for the scaling (3) was calculated for each eigenvalue independently. Subsequently the results of all eigenvalues were superimposed. Such a procedure was mandatory, since the individual mean squared velocities showed large variations. For this reason a scaling with a globally calculated mean squared velocity did not give satisfactory results.

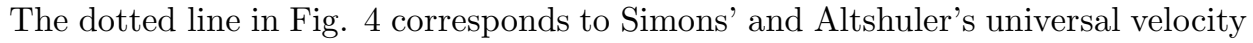
correlation function. Since an analytical expression for this quantity is not available, it was obtained from a random matrix simulation [16]. The overall agreement between experiment and theory is good, apart from some small but significant deviations close to the minimum. It was already mentioned in the beginning that in most studied cases such an agreement was not found. 


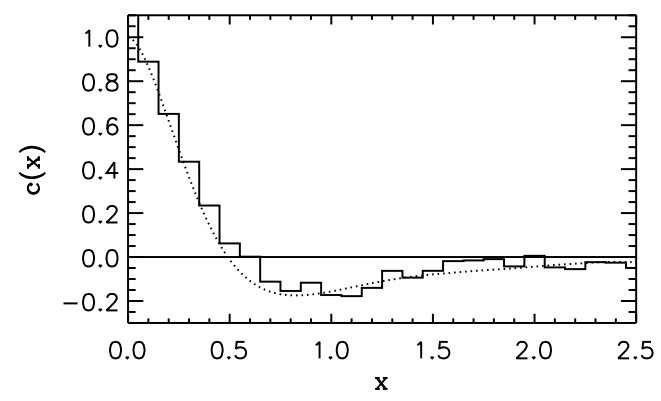

Fig. 4 Velocity autocorrelation function for the quarter Sinai billiard as a function of the position of one length rescaled according to Eq. (3). The dotted line corresponds to Simons' and Altshuler's universal function.

\section{Local level dynamics}

Whether a movable scatterer perturbs the system globally or locally, depends on its size, or, more precisely, on the parameter $\delta=k D$, where $D$ is the diameter of the scatterer and $k$ the wavenumber. For small $\delta$ values $(\delta \ll 1)$ a local level dynamics is expected, whereas with increasing $\delta$ values a gradual transition towards a global behaviour should be observed. Fig. . shows the velocity distribution in the rectangular billiard with randomly distributed scatterers for three different $\delta$ ranges, again in a linear and a logarithmic plot. In Fig. 5(a) the diameter of the movable disk was 4.6 $\mathrm{mm}$, and the eigenvalues were taken in the range 3.4 to $6 \mathrm{GHz}$. In Figs. F(b) and (c) the diameter of the scatterer was $20 \mathrm{~mm}$, and the eigenvalues were in the range 3.4 to $5.8 \mathrm{GHz}$ and 12.5 to $14.3 \mathrm{GHz}$, respectively.

For small $\delta$ values the found velocity distribution is well described by a modified Bessel function

$$
P(v)=\frac{1}{\pi \sqrt{\left\langle v^{2}\right\rangle}} K_{0}\left(\frac{|v|}{\sqrt{\left\langle v^{2}\right\rangle}}\right) .
$$

The logarithmic plot shows that this behaviour is found over two orders of magnitude. Note that now asymptotically a single exponential behaviour is found in contrast to the Gaussian behaviour observed for the global velocities.

With increasing $\delta$ values a transition to Gaussian behaviour is observed as expected. We completed the sequence with a level dynamics in a Sinai billiard, where the central circle was moved, corresponding to $\delta$ values between 30 and 40 . Here the found velocities were again Gaussian distributed (not shown).

A corresponding deviation from the universal behaviour is found for the velocity autocorrelation functions. The left column of Fig. 6 shows the results for the same $\delta$

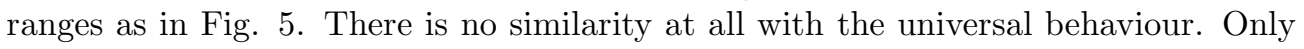
for the largest $\delta$ range the found correlation function seems to approach the universal function.

All these findings are in striking contrast to all what has been expected by the experts. Nevertheless, the discrepancy from the universal behaviour can be quite easily 

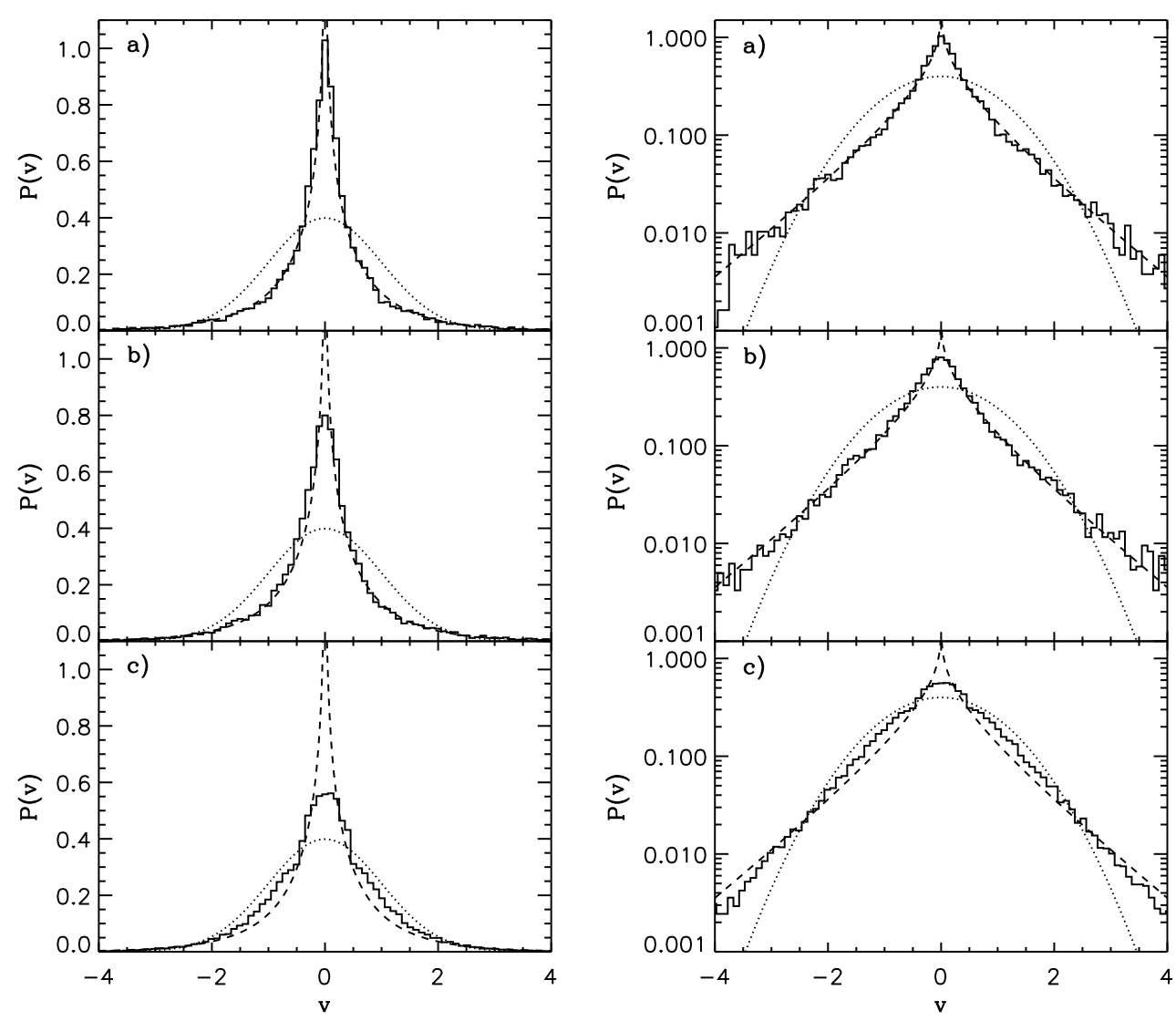

Fig. 5 Velocity distributions in a rectangular billiard with randomly distributed scatterers as a function of the position of one of the scatterers on a linear (left column) and a logarithmic (right column) scale. The histograms were taken in three different ranges of $\delta=k D$, where $D$ is the diameter of the movable scatterer: $0.35<\delta<0.65$ (a), $1.4<\delta<2.6$ (b), $5.1<\delta<5.9$ (c). The dotted line corresponds to a Gaussian, the dashed line to a modified Bessel function.

resolved. It is a well-known fact that the insertion of a metallic object into a quasitwo-dimensional microwave cavity shifts the eigenfrequencies to smaller frequencies. If the dimensions of the perturber are small compared to the wavelength, this shift is proportional to the square of the electric field. This technique has been used routinely for many years to map field distributions in microwave cavities [17] and has recently been applied to the study of wave function in chaotic billiards as well [18, 19, 20]. Applied to the present problem this means that in the limit $\delta \rightarrow 0$ the eigenvalue velocities are given by

$$
\frac{\partial E_{n}}{\partial X}=\alpha \nabla|\psi|^{2}
$$

where $X$ is the position of the scatterer, and $\nabla$ is the gradient taken in the direction of the displacement. $\alpha$ is a constant factor depending exclusively on the geometry of 

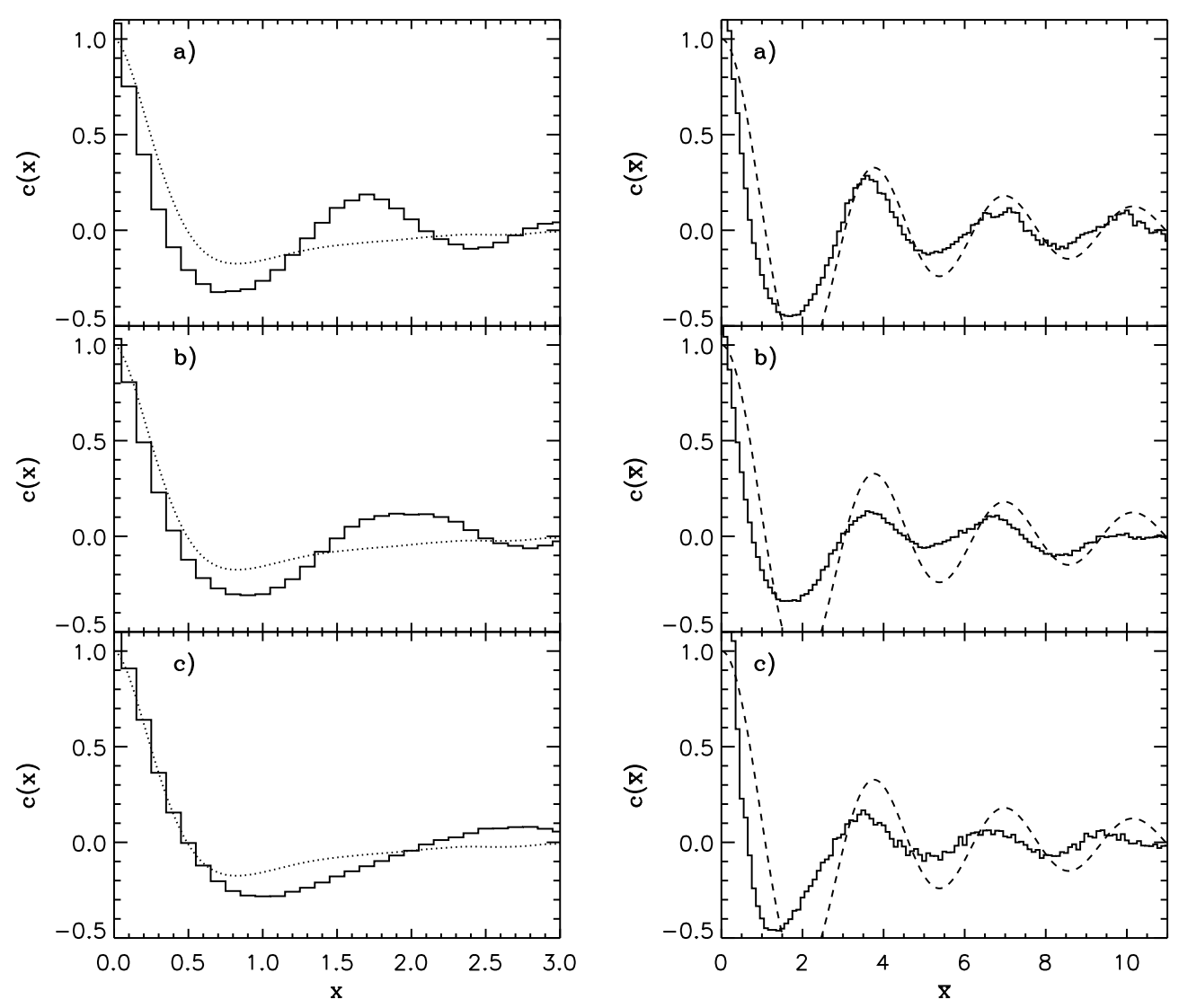

Fig. 6 Velocity autocorrelation functions in a rectangular billiard with randomly distributed scatterers for the same ranges of $\delta$ as in Fig. 5. On the left column the position of the movable scatterer was scaled according to Eq. (3). The dotted line is Simons' and Altshuler's universal function. On the right column the same data are plotted, but with a scaling of the position according to Eq. (10). The dashed lines have been calculated from Eq. (9).

the perturber. It follows for the velocity distribution

$$
P(v)=\langle\delta(v-2 \alpha \psi \nabla \psi)\rangle
$$

where the brackets denote an average of eigenvalues. This average can be calculated, if we assume that the wavefunction $\psi(r)$ can be described by a random superposition of plane waves [21]

$$
\psi(r)=\sum a_{n} e^{i \vec{k}_{n} \vec{r}}
$$

where the modulus of the wavenumbers of all waves contributing is the same, $\left|\vec{k}_{n}\right|=$ $k$, but where amplitudes $a_{n}$ and directions $\vec{k}_{n} / k$ are random. This approach has been proved extremely fruitful in the description of eigenfunctions in chaotic systems [22, 23, 19]. 
By means of the central limit theorem it is now an easy matter to show that under these assmptions $P(\psi)$ and $P(\nabla \psi)$ are uncorrelated and both Gaussian distributed. With these ingredients the average (7) can be calculated, resulting in the modified Bessel function (5) for the velocity distribution. With help of the ansatz (8) the velocity autocorrelation can be calculated as well, using standard techniques 24]. The result is

$$
c(\bar{x})=-\left[J_{0}^{2}(\bar{x})\right]^{\prime \prime}=J_{0}^{2}(\bar{x})-2 J_{1}^{2}(\bar{x})-J_{0}(\bar{x}) J_{2}(\bar{x})
$$

where

$$
\bar{x}=k X .
$$

Note that in contrast to Simons' and Altshuler's approach where the level dynamics parameter $X$ has been scaled by means of the square root of the quadratically averaged velocities, now we have got a straightforward scaling in terms of the wavenumber $k$.

On the right column of Fig. 6 the same data shown on the left column are plotted again, but now with an abscissa scaled according to Eq. (10). The solid line has been calculated from Eq. (9). It describes the experimental results quite well. In particular the wavelengths of the oscillations are reproduced correctly even for the larger $\delta$ ranges. Only an increasing damping of the oscillations with increasing $\delta$ is observed. This oscillatory behaviour in the velocity autocorrelation function, which is already evident from a visual inspection of Fig. 2 (right), is in sharp contrast to the global case, where the autocorrelation function shows only one minimum, while all subsequent oscillations found for the individual levels are completely wiped out by averaging process.

\section{Conclusion}

This work has shown that in contrast to our previous understanding there is not one but there are two regimes of universality. Only for the global case where a small variation of the level dynamics parameter changes the wave functions everywhere, the universal velocity distributions and correlations predicted by Simons and Altshuler are really observed. In this range the scaling of the level dynamics parameter in terms of the square root of the quadratically averaged velocities is the appropriate one.

In the local limit, on the other hand, realized in the present work by moving a small pertuber through a disordered system, another universal regime is observed. Here the scaling of the level dynamics parameter in terms of the wave number is the correct one, and another class of velocity distributions and correlations is found, which has been discussed in this paper.

The issue of this paper has been discussed with numerous colleagues from the mesoscopic community. Discussions with Y. Fyodorov and T. Guhr have been in particular helpful. E. Mucciolo provided us with his unpublished calculation of the universal velocity autocorrelation function. The work has been supported by the Deutsche Forschungsgemeinschaft via the SFB 185 "Nichtlineare Dynamik" as well as by an individual grant. 


\section{References}

[1] J.T. Edwards and D.J. Thouless, J. Phys. C 5 (1972) 807

$2]$ D.J. Thouless, Phys. Rep. 13 (1974) 93

[3] E. Akkermans and G. Montambaux, Phys. Rev. Lett. 68 (1992) 642

[4] A. Szafer and B.L. Altshuler, Phys. Rev. Lett. 70 (1993) 587

[5] B.D. Simons and B.L. Altshuler, Phys. Rev. B 48 (1993) 5422

[6] B.L. Altshuler and B.D. Simons, in Mesoscopic Quantum Physics edited by E. Akkermans et al., North Holland, Amsterdam 1995, p. 1

[7] B.D. Simons et al., Phys. Rev. Lett. 71 (1993) 2899

[8] H. Bruus, C.H. Lewenkopf, and E.R. Mucciolo, Phys. Rev. B 53 (1996) 9968

[9] Y. Hlushchuk et al., to be published.

[10] P. Bertelsen et al., Phys. Rev. Lett. 83 (1999) 2171

[11] M. Barth, U. Kuhl, and H.-J. Stöckmann, Phys. Rev. Lett 82 (1999) 2026

[12] J. Stein, H.-J. Stöckmann, and U. Stoffregen, Phys. Rev. Lett. 75 (1995) 53

[13] P. So et al., Phys. Rev. Lett. 74 (1995) 2662

[14] M. Kollmann et al., Phys. Rev. E 49 (1994) R1

[15] M. Sieber et al., J. Phys. A 28 (1995) 5041

[16] E.R. Mucciolo, private communication

[17] L.C. Maier and J.C. Slater, J. Appl. Phys. 23 (1952) 78

[18] S. Sridhar, Phys. Rev. Lett. 67 (1991) 785

[19] U. Dörr et al., Phys. Rev. Lett. 80 (1998) 1030

[20] D.H. Wu et al., Phys. Rev. Lett. 81 (1998) 2890

[21] M.V. Berry, J. Phys. A 10 (1977) 2083

[22] P. O'Connor, J. Gehlen, and E.J. Heller, Phys. Rev. Lett. 58 (1987) 1296

[23] S.W. McDonald and A.M. Kaufman, Phys. Rev. A 37 (1988) 3067

[24] M. Srednicki and F. Stiernelof, J. Phys. A 29 (1996) 5817 